\title{
Sporadic cases of community acquired legionnaires' disease: an ecological study to identify new sources of contamination
}

\author{
D Che, B Decludt, C Campese, J C Desenclos
}

See end of article for authors' affiliations

.....................

Correspondence to: Dr B Decludt, Institut de Veille Sanitaire,

Département des Maladies Infectieuses, 12 rue du Val d'Osne, 94415 Saint

Maurice cédex, France:

b.decludt@invs.sante.fr

Accepted for publication 14 January 2002

\begin{abstract}
Study objective: To explore the relation between incidence of sporadic and community acquired legionnaires' disease and exposure to potentially contaminated industrial aerosols.

Design: Geographical ecological approach using the postcode as the statistical unit. A multivariate Poisson regression model was used to model the relation between exposure to industrial aerosols and legionnaires' disease.

Setting: Metropolitan France.

Main results: More than 1000 sources of industrial exposure (aerosol and plume of smoke) were identified in 42 French departments. After adjusting for confounding factors, there was a statistically increased incidence of legionnaires' disease in postcodes with plume of smoke in comparison with postcodes without ( $R R=1.45,95 \% \mathrm{Cl}=1.12$ to 1.87$)$, and in postcodes with more than one aerosol in comparison with postcodes without ( $\mathrm{RR}=1.37,95 \% \mathrm{Cl}=1.04$ to 1.79 ).

Conclusion: These findings highlight that any industrial systems generating water aerosols should be regarded as potential sources of contamination for legionnaires' disease.
\end{abstract}

egionnaires' disease is a pneumonia caused by a Gram negative bacillus of the genus Legionella, ${ }^{12}$ which is an Lenvironmental micro-organism found in soil and water. The proportion of pneumonia attributed to Legionella varies from 1\% to $16 \%{ }^{3}$ Many species of Legionella are pathogenic for humans, but most cases (over 90\%) are associated with Legionella pneumophila, with serogroup 1 being the most common. ${ }^{34}$ The incubation period varies from 2 to 10 days. Risk factors for acquisition of legionnaires' disease are well defined. Chronic lung disease, cancer, immunodepression, chronic illnesses, advanced age, and cigarette smoking increase the risk of infection..$^{5-8}$ The case fatality ratio usually does not exceed $10 \%$, but inappropriate treatment or severe underlying illness could be associated with a $40 \%$ case fatality ratio. ${ }^{3}$

Legionnaires' disease can be acquired by the inhalation of aerosol containing Legionella or by microaspiration of contaminated water ${ }^{10}$; person to person transmission has not been described. Many devices generating water aerosols can therefore transmit the infection. The two main known sources of contamination are hot water systems (showers, whirlpool baths) and evaporative cooling systems (cooling towers). ${ }^{11-18}$ Other sources of contamination have been identified: grocery store mist machines, ${ }^{19}$ decorative fountains, ${ }^{20}$ respiratory therapy equipment, ${ }^{21}{ }^{22}$ or industrial aerosol. ${ }^{23} 24$

The link between these sources and legionnaires' disease has been shown mainly by the investigation of disease outbreaks whereas most reported cases are sporadic and community acquired. ${ }^{25}$ Thus, and although studies have shown the link between sporadic cases and sources as cooling towers or home water supplies, ${ }^{76}{ }^{27}$ the source of infection remains unknown for most cases. Before implementing further prevention and control measures, it is important to identify the sources of sporadic cases. Some industrial plants that can spread large volumes of potentially contaminated water aerosols over long distances could be potentially involved in the contamination by Legionella. It has been shown that dissemination of Legionella may occur over long distances (of at least one kilometre).$^{28}$ Furthermore, Legionella can infect and repli- cate within various protozoa found in soil and water. In the environment, protozoa maintain L pneumophila in natural and potable water systems, as they both provide a niche for bacterial replication and serve as a vehicle to protect $L$ pneumophila during the process of water treatment. ${ }^{29}$ As it has been shown that amoebae can be aerosolised,$^{30}$ any device that can spread water aerosols, potentially contaminated by Legionella or amoebae, should be regarded as a possible source of contamination.

The purpose of this study was to explore the relation between sporadic cases of community acquired legionnaires' disease and the exposure to potentially contaminated industrial aerosol.

\section{METHODS}

\section{Study design}

We chose an ecological study, where we seek to estimate risks from data aggregated on a geographical basis. The statistical unit was represented by the residential postcode.

\section{Source of information}

In France, the surveillance of legionnaires' disease was established in 1987 with a mandatory clinician based notification. Cases are reported to local health officers who in turn notify national health authorities (Institut de Veille Sanitaire). The case definition used in this system includes a clinical diagnosis of pneumonia with biological confirmation (culture confirmation, serological tests, direct fluorescent antibody staining, or Legionella urinary antigen test). Information about age, sex, residential postcode, risk factors, and exposure during the 10 days before the onset of the disease are collected. As the notification was changed in 1997 (new case definition with the introduction of Legionella urinary antigen test, new notification card and better collaboration with the information sources as the Centre National de Référence des Legionella, CNR), only the data collected between 1998 and 2000 by the national notification system were used in this study. 
Table 1 Characteristics of the exposed and unexposed postcodes

\begin{tabular}{lll}
\hline & $\begin{array}{l}\text { Exposed postcodes } \\
(\mathrm{n}=661)\end{array}$ & $\begin{array}{l}\text { Unexposed postcodes } \\
(\mathrm{n}=2473)\end{array}$ \\
\hline Size of population (average number of inhabitants) & 20344 & 6838 \\
Number of cases & 204 & 167 \\
Number of person years & 40342926 & 50732955 \\
Rural/urban status & $103(15.6 \%)$ & $1071(43.3 \%)$ \\
$\quad$ Rural & $244(36.9 \%)$ & $690(27.9 \%)$ \\
Urban & $314(47.5 \%)$ & $712(28.8 \%)$ \\
Both & & \\
\hline
\end{tabular}

During 2001, we compiled an inventory of industrial sites that may have been involved in generating potentially contaminated water aerosols during all the three years of the study (1998-2000). Industrial sites with aerosol generating devices need to be either licensed or registered (according to the power of the device) by the relevant authorities. The inventory was prepared in collaboration with the regional administration for industry, research and environment (Direction régionale de l'industrie, de la recherche et de l'environnement, DRIRE), the French ministry of environment, and local public health services. Industrial sites that have been identified as potential source for legionnaires' disease were classified into two groups according to the type of device: producing aerosol (for example, installation using cooling system during an industrial process) or producing plume of smoke (nuclear and thermal power station, incinerator for household refuse).

Demographic information for all statistical units were collected from the Institut National des Statistiques et des Etudes Economiques (INSEE). Metropolitan France is divided in 95 departments, each of them divided into several communes (36 551 in 1990), the smallest administrative unit in France. A postcode includes several communes, except for Paris, Lyon, and Marseille areas, where the communes are divided into different postcodes. Data regarding the number of inhabitants and the urban/rural status were obtained for every postcode included in the analysis. It was not possible to collect data about the age and sex distribution per postcode. Three levels were used for the urban/rural status: "urban", "rural" and "both" for postcodes that included both rural and urban communes. Latitude and longitude data were not available for every postcode. Thus, to take into account a potential geographical gradient, the geographical coordinates of the department were calculated using a scale 1-10 north-south and a 1-10 east-west that were allocated to each postcodes within that department.

\section{Case definition}

A sporadic case of legionnaires' disease was defined as a case that was not part of an identified outbreak.

Patients known to have been hospitalised in the 10 days before onset of disease were assumed to have infection that were possibly nosocomially acquired and were excluded from this study. Patients who have travelled in the 10 days before the onset of disease and for whom the source of exposure has been identified were also excluded from this study.

\section{Statistical analysis}

To explore the relation between legionnaires' disease incidence and exposure to industrial aerosol and/or plume of smoke, a multiple Poisson regression model was used. The covariates were the number of source of exposure per postcode and type of exposure (plume of smoke and/or aerosol), urban/ rural status, and a linear geographical gradient, expressed in terms of latitude and longitude for each department. The choice of whether to include the number of exposure as continuous variables, or one of the sets of strata produced was made by considering likelihood ratio tests, and examination of estimates for each set of strata. Data about latitude and longitude were both included even though one of them might not be statistically significant, in order to maintain a symmetrical expression of the coordinate system.

These data were then used to obtain adjusted relative risks (RR) with 95\% confidence intervals in multivariate Poisson regression model using internal rates. Risks were estimated by using the maximum likelihood method. Interaction effects were considered between latitude and longitude. These analyses were performed using Egret software. ${ }^{31}$

The standardised incidence ratio (SIR), the ratio of the observed to the expected number of incident cases were also used to estimate the risk of legionnaires' disease for the different exposure categories. The expected number of cases was calculated by multiplying the observed number of person years in each postcode by the legionnaires' disease incidence derived from the entire French population.

The SIRs (based on external comparisons) were similar to the RRs from Poisson regression (with internal rates), indicating that the studied cohort was representative of the general population (data not shown).

\section{RESULTS}

\section{Number and characteristics of cases}

During the years 1998-2000, 880 sporadic cases of community acquired legionnaires' disease were notified to the Institut de Veille Sanitaire. The male to female sex ratio was 3.3 (671 men and 205 women). The average age was 57.4, but women were statistically older $(61.2$ years old versus $56.3, \mathrm{p}<0.001)$. The case fatality ratio was $14 \%$ (120/855). Risk factors had been identified for 611 patients (69\%) with cigarette smoking the most common (43\%). The Legionella species was identified for 760 patients and Legionella pneumophila 1 was identified from 604 patients $(79 \%)$.

\section{Number and characteristics of exposure}

Data for exposure to aerosol and plume of smoke were available for 42 French departments (44\%), in which 1135 sources of exposure were identified, including 1015 sources of aerosols and 120 sources of plume of smoke.

\section{Description of the geographical units}

A total of 3134 postcodes in the 42 French departments, representing 53\% of the entire French metropolitan population, were therefore included in the analysis. The average number of inhabitants per postcode was 9687 and for comparative purposes, in France in 1990, the average was 9485. Within these postcodes, 371 sporadic cases of community acquired legionnaires' disease were identified. For 661 postcodes $(21.1 \%)$, we recorded at least one source of exposure (table 1 ). For each postcode, the number of source of exposure varies from 0 to 9 for the aerosol and from 0 to 2 for the plume of smoke (only one postcode with two plumes of smoke); 236 postcodes $(36 \%)$ were exposed to more than one source. Within the exposed postcodes, there was more urban (36.9\%) than rural postcodes $(15.6 \%)(\mathrm{p}<0.01)$. 


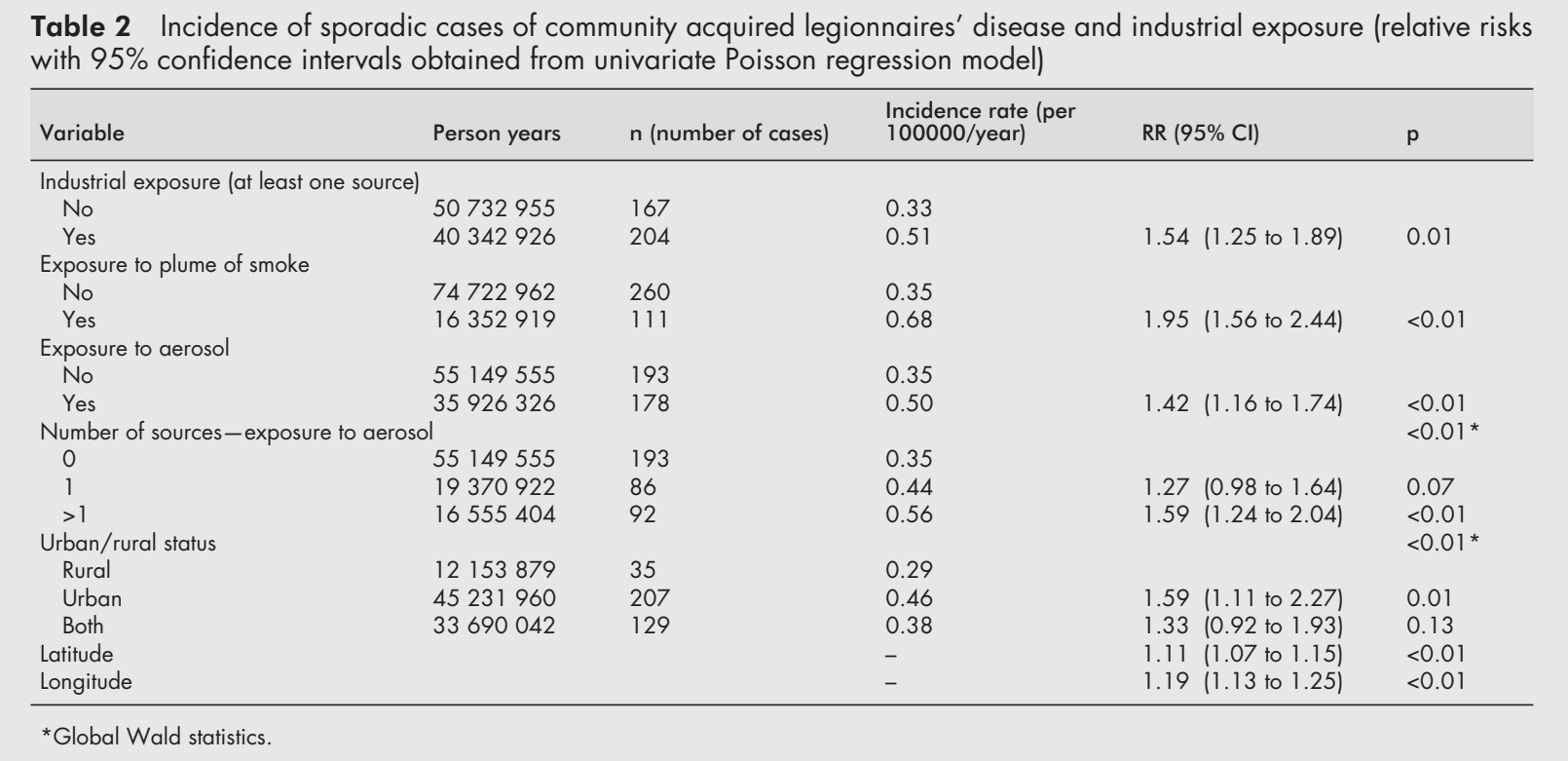

Table 3 Incidence of sporadic cases of community acquired legionnaires' disease and industrial exposure (relative risks with $95 \%$ confidence intervals obtained from multivariate Poisson regression model)

\begin{tabular}{lll}
\hline Variable & RR $(95 \% \mathrm{cl})$ & $\mathrm{p}$ \\
\hline Plume of smoke & $1.45(1.12$ to 1.87$)$ & $<0.01$ \\
Number of aerosols & - & 0.07 \\
0 & $1.0($ ref $)$ & \\
1 & $1.06(0.81$ to 1.38$)$ & 0.68 \\
$\geqslant 2$ & $1.37(1.04$ to 1.79$)$ & 0.02 \\
Latitude & $1.37(1.18$ to 1.58$)$ & $<0.01$ \\
Longitude & $1.42(1.25$ to 1.61$)$ & $<0.01$ \\
Latitude $\times$ longitude & $0.97(0.95$ to 0.99$)$ & $<0.01$ \\
\hline
\end{tabular}

\section{Univariate analysis}

The univariate analysis showed that all variables were statistically associated with the disease (table 2). The unadjusted RR for the exposure to aerosol or plume of smoke (at least one source of exposure) was 1.54 (95\% CI $=1.25$ to 1.89 ), however, when the exposure to aerosol is distinguished from the exposure to plume of smoke, we obtain a greater risk for exposure to plume of smoke $(\mathrm{RR}=1.95,95 \% \mathrm{CI}=1.56$ to 2.44$)$ than to aerosol $(\mathrm{RR}=1.42,95 \% \mathrm{CI}=1.16$ to 1.74$)$. These two variables were therefore included separately in the multivariate model.

\section{Multivariate analysis}

In the final multivariate model we did not keep the urban/rural status, which was not statistically associated with the incidence of disease $(p=0.58)$. The exposure to plume of smoke was significantly associated with the incidence of disease $(\mathrm{RR}=1.45,95 \% \mathrm{CI}=1.12$ to $1.87, \mathrm{p}<0.01)$ (table 3$)$. For the exposure to aerosol, we found no significant relation $(\mathrm{p}=0.07)$. On the other hand, there is a positive relation between incidence and exposure to more than one source of aerosol $(\mathrm{RR}=1.37 ; 95 \% \mathrm{CI}=1.04$ to $1.79, \mathrm{p}=0.02)$. Both latitude and longitude were positively associated with the incidence of disease in the final model.

\section{DISCUSSION}

Our study showed that exposure to industrial plume of smoke is statistically associated with the incidence of legionnaires' disease $(\mathrm{RR}=1.45,95 \% \mathrm{CI}=1.12$ to 1.87$)$. Moreover, the exposure to more than one source of aerosol was also associated with the incidence of disease $(\mathrm{RR}=1.37 ; 95 \% \mathrm{CI}=1.04$ to 1.79). As the availability of data was at the postcode level, we used an ecological study design to explore the relation between exposure and incidence of legionnaires' disease. This type of study, despite its methodological limitations (see below), offers many advantages: low cost and convenience, appropriateness for environmental exposure and simplicity of analysis and presentation. ${ }^{32}$ As we could not measure relevant exposure at the individual level, the only practical way to assess the exposure was ecologically. Poisson regression is more and more used for ecological analysis (particularly for cancer epidemiology), but for infectious diseases, this method is often not appropriate because of the non-independence of events due to person to person transmission. For legionnaires' disease, such transmission does not exist ${ }^{1}$ and this regression method was then appropriate to apply.

Nevertheless, some methodical limitations must be acknowledged of this study design and particularly that of ecological bias (or ecological fallacy or aggregation bias), which represents the failure of expected ecological effect estimates to reflect the effect at the individual level. ${ }^{32-34}$ A potential strategy for minimising ecological bias is to use smaller units in order to make the groups more homogeneous with respect to the exposure. ${ }^{32}$ For this reason, we used the postcode as the geographical unit, which was the smallest uniform geographical unit we can use in France.

In France, the notification of legionnaires' disease is not complete. A capture-recapture analysis conducted in France in 1998 has estimated that only $30 \%$ of cases were notified. ${ }^{35}$ As the notification is clinician based and as the capture-recapture analysis have not highlighted differences for the level of notification between Paris area, Rhône Alpes, or the rest of metropolitan France, whereas an industrialisation heterogeneity exist, we assumed that the under-notification is not related to the exposure status (non-differential misclassification). Moreover, the relation between industrial exposure (plume of smoke or aerosol) and sporadic cases of community acquired legionnaires' disease having not been described yet, the presence of a plausible source known to local clinicians should not modify their reporting to local health authorities. In the same way, the inventory of sources of exposure is certainly not exhaustive and some postcodes may have been wrongly ascribed unexposed. As this classification error is probably not related to the incidence of legionnaires' disease, we can 
assume that the exposure misclassification is non-differential. In our study, as the exposure of groups is characterised by a single common measure (and not by a combination of individual observations, for example, proportion of people exposed) these types of misclassification (non-differential) should tend to attenuate the relation between exposure and disease. ${ }^{36}$

Because of the lack of aggregated data at the postcode level, some data were not integrated in our study. For example, it was not possible to take the individual risk factors into account. To do so, it would have been necessary to collect data about the proportion of men and women, smokers, immunodepression, or cancer, and about the age distribution for all postcodes, but these data were not available. For the same reason, data about wind (strength and direction) or about altitude of industries, which can influence the spread of potentially contaminated water and therefore, the exposed or unexposed status, were not included in this study. For all these reasons, we have introduced a geographical gradient in the analysis. In fact, the introduction of a geographical gradient in a multiple Poisson regression model reduced strong spatial correlation, but can also be considered as a proxy confounding variable. ${ }^{37}$

We have also made the assumption that population was stable over time. The small number of cases compared with any change of the population over time greatly minimises this problem. In the same way, we did not take the migration bias into account. This bias can affect the validity of ecological study essentially for long latency chronic disease ${ }^{38}$ and will probably not apply for legionnaires' disease for which the incubation period is between 2 to 10 days.

Despite the methodological limitations that we have discussed above, the results of our exploratory study suggest that legionnaires' disease may be transmitted through aerosols generated by industrial devices. These preliminary findings need to be confirmed for entire metropolitan France to assure the representativeness of the data. Analytical studies (case-control studies in particular) at an individual level will have to assess the role of such exposure and determine its aetiological fraction. For that, data about age, sex, and other individual risk factors (cancer, chronic illness ....) will have to be collected. Geographical studies on a national scale will also have to be performed to confirm the geographical gradient, and complementary studies should explain it if necessary. Finally, community outbreaks should also be used to assess the role of industrial exposures in the transmission of Legionella. If these studies confirm our results, then the question of control measures and of the collection of this information on the case investigation will need to be discussed.

\section{Authors' affiliations}

D Che, B Decludt, C Campese, J C Desenclos, Institut de Veille Sanitaire, Saint Maurice, France

Funding: this work was supported by the Fondation pour la Recherche Médicale, France.

Conflicts of interest: none.

\section{REFERENCES}

1 Fraser DW, Tsai TR, Orenstein W, et al. Legionnaires' disease: description of an epidemic of pneumonia. N Engl J Med 1977:297: 1189-97.

2 McDade JE, Shepard CC, Fraser DW, et al. Legionnaires' disease: isolation of a bacterium and demonstration of its role in other respiratory disease. N Engl J Med 1977;297:1 197-203.

3 Muder RR, Yu VL, Fang GD. Community-acquired Legionnaires' disease. Semin Respir Infect 1989:4:32-9.

4 Campese C, Decludt B. Les légionelloses déclarées en France en 1998. Bull Epidemiol Hebd 2000;12:49-51.
5 England AC, III, Fraser DW, Plikaytis BD, et al. Sporadic legionellosis in the United States: the first thousand cases. Ann Intern Med 1981;94:164-70.

6 Marston BJ, Lipman HB, Breiman RF. Surveillance for Legionnaires' disease. Risk factors for morbidity and mortality. Arch Intern Med 1994; 154:2417-22

7 Storch G, Baine WB, Fraser DW, et al. Sporadic community-acquired Legionnaires' disease in the United States. A case-control study. Ann Intern Med 1979:90:596-600.

8 Stout JE, Yu VL. Legionellosis. N Engl J Med 1997;337:682-7.

9 Muder RR, Yu VL, Woo AH. Mode of transmission of Legionella pneumophila. A critical review. Arch Intern Med 1986;146:1607-12

10 Edelstein PH. Legionnaires' disease. Clin Infect Dis 1993;16:741-7.

11 Decludt B, Guillotin L, Van Gastel B, et al. Foyer épidémique de légionellose à Paris en juin 1998. Bull Epidemiol Hebd 1999;21:83-5.

12 Dondero TJ, Jr, Rendtorff RC, Mallison GF, et al. An outbreak of Legionnaires' disease associated with a contaminated air- conditioning cooling tower. N Engl J Med 1980;302:365-70.

13 Fraser DW. Potable water as a source for legionellosis. Environ Health Perspect 1985;62:337-41.

14 Garbe PL, Davis BJ, Weisfeld JS, et al. Nosocomial Legionnaires' disease. Epidemiologic demonstration of cooling towers as a source. JAMA 1985;254:521-4.

15 McEvoy M, Batchelor N, Hamilton G, et al. A cluster of cases of legionnaires' disease associated with exposure to a spa pool on display. Commun Dis Public Health 2000;3:43-5.

16 Miller RP. Cooling towers and evaporative condensers. Ann Intern Med 1979:90:667-70.

17 Stout JE, Yu VL, Muraca P, et al. Potable water as a cause of sporadic cases of community-acquired legionnaires' disease. N Engl J Med 1992;326:151-5.

18 Straus WL, Plouffe JF, File TM, et al. Risk factors for domestic acquisition of legionnaires disease. Ohio legionnaires Disease Group. Arch Intern Med 1996:156:1685-92.

19 Mahoney FJ, Hoge CW, Farley TA, et al. Communitywide outbreak of Legionnaires' disease associated with a grocery store mist machine. J Infect Dis 1992;165:736-9.

20 Hlady WG, Mullen RC, Mintz CS, et al. Outbreak of Legionnaire's disease linked to a decorative fountain by molecular epidemiology. Am Epidemiol 1993;138:555-62.

21 Arnow PM, Chou T, Weil D, et al. Nosocomial Legionnaires' disease caused by aerosolized tap water from respiratory devices. J Infect Dis 1982;146:460-7.

22 Mastro TD, Fields BS, Breiman RF, et al. Nosocomial Legionnaires' disease and use of medication nebulizers. J Infect Dis 1991;163:66771

23 From the Centers for Disease Control and Prevention. Outbreak of Legionnaires' disease among automotive plant workers-Ohio, 2001. JAMA 2001;285:2848-9.

24 Allen KW, Prempeh H, Osman MS. Legionella pneumonia from a novel industrial aerosol. Commun Dis Public Health 1999;2:294-6.

25 Campese C, Decludt B. Les légionelloses déclarées en France en 1999. Bull Epidemiol Hebd 2000:52:235-7.

26 Bhopal R. Source of infection for sporadic Legionnaires' disease: a review. J Infect 1995;30:9-12.

27 Bhopal RS, Fallon RI, Buist EC, et al. Proximity of the home to a cooling tower and risk of non-outbreak Legionnaires' disease. BM 1991;302:378-83.

28 Addiss DG, Davis JP, LaVenture $M$, et al. Community-acquired Legionnaires' disease associated with a cooling tower: evidence for longer-distance transport of Legionella pneumophila. Am J Epidemiol 1989; 130:557-68

29 Barbaree JM, Fields BS, Feeley JC, et al. Isolation of protozoa from water associated with a legionellosis outbreak and demonstration of intracellular multiplication of Legionella pneumophila. Appl Environ Microbiol 1986;51:422-4.

30 Brieland JK, Fantone JC, Remick DG, et al. The role of Legionella pneumophila-infected Hartmannella vermiformis as an infectious particle in a murine model of Legionnaire's disease. Infect Immun 1997;65:5330-3

31 Egret. Software. Seattle: Egret, statistics and epidemiology research corporation, 1990.

32 Morgenstern H. Ecologic studies in epidemiology: concepts, principles, and methods. Annu Rev Public Health 1995;16:61-81.

33 Greenland S, Morgenstern H. Ecological bias, confounding, and effect modification. Int J Epidemiol 1989;18:269-74.

34 Morgenstern $\mathbf{H}$. Uses of ecologic analysis in epidemiologic research. Am J Public Health 1982;72:1336-44.

35 Nardone A, Decludt B. Evaluation épidémiologique du système de surveillance de la légionellose en France en 1998. Saint Maurice: Institut de Veille Sanitaire, 2000.

36 Brenner H, Savitz DA, Jockel KH, et al. Effects of nondifferential exposure misclassification in ecologic studies. Am J Epidemiol $1992 ; 135: 85-95$

37 Richardson S. A method for testing the significance of geographical correlations with application to industrial lung cancer in France. Stat Med 1990;9:515-28.

38 Tong S. Migration bias in ecologic studies. Eur J Epidemiol 2000; 16:365-9. 